\title{
Positive Periodic Solution for Second-Order Singular Semipositone Differential Equations
}

\author{
Xiumei Xing \\ School of Mathematics and Statistics, Yili Normal University, Yining City 835000, China \\ Correspondence should be addressed to Xiumei Xing; xingxm09@163.com
}

Received 7 November 2012; Accepted 20 December 2012

Academic Editor: Jifeng Chu

Copyright (C) 2013 Xiumei Xing. This is an open access article distributed under the Creative Commons Attribution License, which permits unrestricted use, distribution, and reproduction in any medium, provided the original work is properly cited.

We study the existence of a positive periodic solution for second-order singular semipositone differential equation by a nonlinear alternative principle of Leray-Schauder. Truncation plays an important role in the analysis of the uniform positive lower bound for all the solutions of the equation. Recent results in the literature (Chu et al., 2010) are generalized.

\section{Introduction}

In this paper, we study the existence of positive $T$-periodic solutions for the following singular semipositone differential equation:

$$
x^{\prime \prime}+h(t) x^{\prime}+a(t) x=f\left(t, x, x^{\prime}\right),
$$

where $h, a \in C(R / T Z, R)$ and the nonlinearity $f \in C((R / T Z) \times$ $(0,+\infty) \times R, R)$ satisfies $f\left(t, x, x^{\prime}\right) \geq-M$ for some $M>0$. In particular, the nonlinearity may have a repulsive singularity at $x=0$, which means that

$$
\lim _{x \rightarrow 0^{+}} f(t, x, y)=+\infty, \quad \text { uniformly in }(t, y) \in R^{2} .
$$

Electrostatic or gravitational forces are the most important examples of singular interactions.

During the last two decades, the study of the existence of periodic solutions for singular differential equations has attracted the attention of many researchers [1-4]. Some strong force conditions introduced by Gordon [5] are standard in the related earlier works $[6,7]$. Compared with the case of a strong singularity, the study of the existence of periodic solutions under the presence of a weak singularity is more recent $[2,8,9]$, but has also attracted many researchers. Some classical tools have been used to study singular differential equations in the literature, including the method of upper and lower solutions [10], degree theory [11], some fixed point theorem in cones for completely continuous operators
[12], Schauder's fixed point theorem $[8,9,13]$, and a nonlinear Leray-Schauder alternative principle $[2,3,14,15]$.

However the singular differential equations, in which there is the damping term, that is, the nonlinearity is dependent on the derivative, has not attracted much attention in the literature. Several existence results can be found in [14, 16, 17].

The aim of this paper is to further show that the nonlinear Leray-Schauder alternative principle can be applied to (1) in the semipositone cases, that is, $f\left(t, x, x^{\prime}\right) \geq-M$ for some $M>0$.

The remainder of the paper is organized as follows. In Section 2, we state some known results. In Section 3, the main results of this paper are stated and proved. To illustrate our result, we select the following system:

$$
x^{\prime \prime}+h(t) x^{\prime}+a(t) x=\left(1+|x|^{\gamma}\right)\left(x^{-\alpha}+\mu x^{\beta}\right)+e(t),
$$

where $\alpha>1, \beta>0,1>\gamma \geq 0, \mu>0$ is a positive parameter, $e(t)$ is a $T$-periodic function.

In this paper, let us fix some notations to be used in the following: given $\varphi \in L^{1}[0, T]$, we write $\varphi>0$ if $\varphi \geq 0$ for almost everywhere $t \in[0, T]$ and it is positive in a set of positive measure. The usual $L^{p}$-norm is denoted by $\|\cdot\|_{p}$. $p^{*}$ and $p_{*}$ the essential supremum and infinum of a given function $p \in L^{1}[0, T]$, if they exist. 


\section{Preliminaries}

We say that

$$
x^{\prime \prime}+h(t) x^{\prime}+a(t) x=0
$$

associated to the periodic boundary conditions

$$
x(0)=x(T), \quad x^{\prime}(0)=x^{\prime}(T),
$$

is nonresonant when its unique solutions is the trival one. When (4)-(5) is nonresonant, as a consequence of Fredholm's alternative, the nonhomogeneous equation

$$
x^{\prime \prime}+h(t) x^{\prime}+a(t) x=l(t)
$$

admits a unique $T$-periodic solution, which can be written as

$$
x(t)=\int_{0}^{T} G(t, s) l(s) d s,
$$

where $G(t, s)$ is the Green's function of problem (4)-(5). Throughout this paper, we assume that the following standing hypothesis is satisfied.

(A) The Green function $G(t, s)$, associated with (4)-(5), is positive for all $(t, s) \in[0, T] \times[0, T]$.

In other words, the strict antimaximum principle holds for (4)-(5).

Definition 1. We say that (4) admits the antimaximum principle if (6) has a unique $T$-periodic solution for any $l \in$ $\mathbb{C}(\mathbb{R} / T \mathbb{Z})$ and the unique $T$-periodic solution $x_{l}(t)>0$ for all $t$ if $l>0$.

Under hypothesis (A), we denote

$$
A=\min _{0 \leq s, t \leq T} G(t, s), \quad B=\max _{0 \leq s, t \leq T} G(t, s), \quad \iota=\frac{A}{B} .
$$

Thus $B>A>0$ and $0<\iota<1$. We also use $w(t)$ to denote the unique periodic solution of $(6)$ with $l(t)=1$ under condition (5), that is, $w(t)=(\mathscr{L} 1)(t)$. In particular, $T A \leq w(t) \leq T B$.

With the help of $[18,19]$, the authors give a sufficient condition to ensure that (4) admits the antimaximum principle in [14]. In order to state this result, let us define the functions

$$
\begin{gathered}
\sigma(h)(t)=\exp \left(\int_{0}^{t} h(s) d s\right), \\
\sigma_{1}(h)(t)=\sigma(h)(T) \int_{0}^{t} \sigma h(s) d s+\int_{t}^{T} \sigma(h)(s) d s .
\end{gathered}
$$

Lemma 2 (see [14, Corollary 2.6]). Assume that $a \neq \equiv 0$ and the following two inequalities are satisfied:

$$
\begin{gathered}
\int_{0}^{T} a(s) \sigma(h)(s) \sigma_{1}(-h)(s) d s \geq 0, \\
\sup _{0 \leq t \leq T}\left\{\int_{t}^{t+T} \sigma(-h)(s) d s \int_{t}^{t+T}[a(s)]_{+} \sigma(h)(s) d s\right\} \leq 4,
\end{gathered}
$$

where $[a(s)]_{+}=\max \{a(s), 0\}$. Then the Green's function $G(t, s)$, associated with (5), is positive for all $(t, s) \in[0, T] \times[0, T]$.
Next, recall a well-known nonlinear alternative principle of Leray-Schauder, which can be found in [20] and has been used by Meehan and O'Regan in [4].

Lemma 3. Assume $\Omega$ is an open subset of a convex set $K$ in a normed linear space $X$ and $p \in \Omega$. Let $T: \bar{\Omega} \rightarrow K$ be a compact and continuous map. Then one of the following two conclusions holds:

(I) $T$ has at least one fixed point in $\bar{\Omega}$.

(II) There exists $x \in \partial \Omega$ and $0<\lambda<1$ such that $x=$ $\lambda T x+(1-\lambda) p$.

In applications below, we take $K=C_{T}^{1}=\left\{x: x, x^{\prime} \epsilon\right.$ $C(R / T Z, R)\} \subset X$ with the norm $\|x\|=\max _{t \in[0, T]}|x(t)|$ and define $\Omega=\left\{x \in C_{T}^{1}:\|x\|<r\right\}$.

\section{Main Results}

In this section, we prove a new existence result of (1).

Theorem 4. Suppose that (4) satisfies (A) and

$$
a(t)>0 .
$$

Furthermore, assume that there exist three constants $M, R_{0}, r>$ $M w^{*} / \iota$ such that:

$\left(\mathrm{H}_{1}\right) F(t, x, y)=f(t, x, y)+M \geq 0$ for all $(t, x, y) \in[0, T] \times$ $(0, r] \times R$

$\left(\mathrm{H}_{2}\right) f(t, x, y) \geq g_{0}(x)$ for $(t, x, y) \in[0, T] \times\left(0, R_{0}\right] \times R$, where the nonincreasing continuous function $g_{0}(x)>0$ satisfies $\lim _{x \rightarrow 0^{+}} g_{0}(x)=+\infty$ and $\lim _{x \rightarrow 0^{+}} \int_{x}^{R_{0}} g_{0}(u) d u=+\infty$.

$\left(\mathrm{H}_{3}\right) 0 \leq F(t, x, y) \leq(g(x)+h(x)) \varrho(|y|)$, for all $(t, x, y) \in$ $[0, T] \times(0, r] \times R$, where $g(\cdot)>0$ is nonincreasing in $(0, r]$ and $h(\cdot) / g(\cdot) \geq 0, \varrho(\cdot) \geq 0$ are nondecreasing in $(0, r]$.

$\left(\mathrm{H}_{4}\right)$

$$
\frac{r}{g\left(\iota r-M w^{*}\right)(1+h(r) / g(r)) \varrho((r+M) L)}>w^{*},
$$

where

$$
L=\frac{2 \int_{0}^{T} a(t) \sigma(h)(t) d t}{\min _{0 \leq t \leq T} \sigma(h)(t)}
$$

Then (1) has at least one positive periodic solution $u(t)$ with $0<\|u+M w\| \leq r$.

Proof. For convinence, let us write $Z(t)=x(t)-M w(t)$, $Z_{n}(t)=x_{n}(t)-M w(t)$, where $w(t)=(\mathscr{L} 1)(t)$. Let

$$
A_{h}=\min _{0 \leq t \leq T} \sigma(h)(t), \quad B_{h}=\max _{0 \leq t \leq T} \sigma(h)(t), \quad \iota_{h}=\frac{B_{h}}{A_{h}},
$$

$$
\bar{M}_{h}=(r+M) L \cdot \max _{0 \leq t \leq T}|h(t)| .
$$


First we show that

$$
x^{\prime \prime}+h(t) x^{\prime}+a(t) x=F\left(t, Z(t), Z^{\prime}(t)\right)
$$

has a solution $x$ satisfying (5), $0<\|x\| \leq r$ and $Z(t)>0$ for $t \in[0, T]$. If this is true, it is easy to see that $Z(t)$ will be a positive solution of (1)-(5) with $0<\|Z+M w\| \leq r$.

Choose $n_{0} \in\{1,2, \ldots\}$ such that $1 / n_{0}<r$, and then let $N_{0}=\left\{n_{0}, n_{0}+1+\cdots\right\}$

Consider the family of equations

$$
x^{\prime \prime}+h(t) x^{\prime}+a(t) x=\lambda F_{n}\left(t, Z(t), Z^{\prime}(t)\right)+\frac{a(t)}{n},
$$

where $\lambda \in[0,1], n \in N_{0}, x \in B_{r}=\{x:\|x\|<r\}$ and $F_{n}(t, x, y)=F(t, \max \{1 / n, x\}, y)$.

A $T$-periodic solution of (17) is just a fixed of the operator equation

$$
x=\lambda T_{n}(x)+(1-\lambda) p,
$$

where $p=1 / n$ and $T_{n}$ is a completely continuous operator defined by

$$
\left(T_{n} x\right)(t)=\int_{0}^{T} G(t, s) F_{n}\left(s, Z(s), Z^{\prime}(s)\right) d s+\frac{1}{n},
$$

where we have used the fact

$$
\int_{0}^{T} G(t, s) a(s) d s \equiv 1
$$

We claim that for any $T$-periodic solution $x_{n}(t)$ of (17) satisfies

$$
\left\|x_{n}^{\prime}\right\| \leq L r
$$

Note that the solution $x_{n}(t)$ of (17) is also satisfies the following equivalent equation

$$
\begin{aligned}
& \left(\sigma(h)(t) x_{n}^{\prime}\right)^{\prime}+a(t) \sigma(h)(t) x_{n} \\
& \quad=\sigma(h)(t)\left(\lambda F_{n}\left(t, Z_{n}(t), Z_{n}^{\prime}(t)\right)+\frac{a(t)}{n}\right) .
\end{aligned}
$$

Integrating (22) from 0 to $T$, we obtain

$$
\begin{aligned}
& \int_{0}^{T} a(t) \sigma(h)(t) x_{n}(t) d t \\
& \quad=\int_{0}^{T} \sigma(h)(t)\left(\lambda F_{n}\left(t, Z_{n}(t), Z_{n}^{\prime}(t)\right)+\frac{a(t)}{n}\right) d t .
\end{aligned}
$$

By the periodic boundary conditions, we have $x^{\prime}\left(t_{0}\right)=0$ for some $t_{0} \in[0, T]$. Therefore,

$$
\begin{aligned}
\mid \sigma & (h)(t) x_{n}^{\prime}(t) \mid \\
& =\left|\int_{t_{0}}^{t}\left(\sigma(h)(s) x_{n}^{\prime}(s)\right)^{\prime} d s\right| \\
& =\left|\int_{t_{0}}^{t} \sigma(h)(s)\left(\lambda F_{n}\left(s, Z_{n}(s), Z_{n}^{\prime}(s)\right)+\frac{a(s)}{n}-a(s) x_{n}(s)\right)\right| d s \\
& \leq\left|\int_{0}^{T} \sigma(h)(s)\left(\lambda F_{n}\left(s, Z_{n}(s), Z_{n}^{\prime}(s)\right)+\frac{a(s)}{n}+a(s) x_{n}(s)\right)\right| d s \\
& =2 \int_{0}^{T} \sigma(h)(s) a(s) x_{n}(s) d s \\
& \leq 2 r \int_{0}^{T} \sigma(h)(s) a(s) d s,
\end{aligned}
$$

where we have used the assumption (11) and $\left\|x_{n}\right\|<r$. Therefore,

$$
\left(\min _{0 \leq t \leq T} \sigma(h)(t)\right)\left|x_{n}^{\prime}(t)\right| \leq 2 r \int_{0}^{T} \sigma(h)(s) a(s) d s,
$$

which implies that (21) holds. In particular, let $\lambda F_{n}(t, Z(t)$, $\left.Z^{\prime}(t)\right)+a(t) / n=1$ in (17), we have

$$
\left\|w^{\prime}(t)\right\| \leq L \text {. }
$$

Choose $n_{1} \in N_{0}$ such that $1 / n_{1} \leq R_{1}$, and then let $N_{1}=$ $\left\{n_{1}, n_{1}+1, \ldots\right\}$. The following lemma holds.

Lemma 5. There exists an integer $n_{2}>n_{1}$ large enough such that, for all $n \in N_{2}=\left\{n_{2}, n_{2}+1, \ldots\right\}$,

$$
Z_{n}(t)=x_{n}(t)-M w(t) \geq \frac{1}{n} \text {. }
$$

Proof. The lower bound in (27) is established by using the strong force condition of $f(t, x, y)$. By condition $\left(\mathrm{H}_{2}\right)$, there exists $R_{1} \in\left(0, R_{0}\right)$ and a continuous function $\widetilde{g}_{0}(x)$ such that

$$
F(t, x, y)-a(t) x \geq \tilde{g}_{0}(x)>\max \left\{M+\bar{M}, \iota_{h} r\|a\|_{1}\right\}
$$

for all $(t, x, y) \in[0, T] \times\left(0, R_{1}\right] \times R$, where $\tilde{g}_{0}(x)$ satisfies also the strong force condition like in $\left(\mathrm{H}_{2}\right)$.

For $n \in N_{1}$, let $\alpha_{n}=\min _{0 \leq t \leq T} Z_{n}(t), \beta_{n}=\max _{0 \leq t \leq T} Z_{n}(t)$. If $\alpha_{n} \geq R_{1}$, due to $n \in N_{1}$, (27) holds.

If $\alpha_{n}<R_{1}$, we claim that, for all $n \in N_{1}$,

$$
\beta_{n}>R_{1} \text {. }
$$

Otherwise, suppose that $\beta_{n} \leq R_{1}$ for some $n \in N_{1}$. Then it is easy to verify

$$
F_{n}\left(t, Z_{n}(t), Z_{n}^{\prime}(t)\right)>\iota_{h} r\|a\|_{1} .
$$

In fact, if $1 / n \leq Z_{n}(t) \leq R_{1}$, we obtain from (28)

$$
\begin{aligned}
F_{n}\left(t, Z_{n}(t), Z_{n}^{\prime}(t)\right) & =F\left(t, Z_{n}(t), Z_{n}^{\prime}(t)\right) \\
& \geq a(t) Z_{n}(t)+\tilde{g}_{0}\left(Z_{n}(t)\right) \\
& \geq \tilde{g}_{0}\left(Z_{n}(t)\right) \\
& >\iota_{h} r\|a\|_{1} .
\end{aligned}
$$


and, if $Z_{n}(t) \leq 1 / n$, we have

$$
\begin{aligned}
F_{n}\left(t, Z_{n}(t), Z_{n}^{\prime}(t)\right) & =F\left(t, \frac{1}{n}, Z_{n}^{\prime}(t)\right) \geq \frac{a(t)}{n}+\tilde{g}_{0}\left(\frac{1}{n}\right) \\
& \geq \tilde{g}_{0}\left(\frac{1}{n}\right)>\iota_{h} r\|a\|_{1} .
\end{aligned}
$$

Integrating (22) (with $\lambda=1$ ) from 0 to $T$, we deduce that

$$
\begin{aligned}
0= & \int_{0}^{T}\left\{\left(\sigma(h)(t) x_{n}^{\prime}\right)^{\prime}+a(t) \sigma(h)(t) x_{n}\right. \\
& \left.\quad-\sigma(h)(t)\left(F_{n}\left(t, Z_{n}(t), Z_{n}^{\prime}(t)\right)+\frac{a(t)}{n}\right)\right\} d t \\
= & \int_{0}^{T} a(t) \sigma(h)(t) x_{n} d t \\
& -\int_{0}^{T} \sigma(h)(t) F_{n}\left(t, Z_{n}(t), Z_{n}^{\prime}(t)\right) d t \\
& -\int_{0}^{T} \sigma(h)(t) \frac{a(t)}{n} d t \\
< & \int_{0}^{T} a(t) \sigma(h)(t) x_{n} d t \\
& -\int_{0}^{T} \sigma(h)(t) F_{n}\left(t, Z_{n}(t), Z_{n}^{\prime}(t)\right) d t
\end{aligned}
$$

$<0$,

where estimation (30) and the fact $\left\|x_{n}\right\|<r$ are used. This is a contradiction. Hence (29) holds.

Due to $\alpha_{n}<R_{1}$, that is, $\alpha_{n}=\min _{0 \leq t \leq T}\left[x_{n}(t)-M w(t)\right]=$ $x_{n}\left(a_{n}\right)-M w\left(a_{n}\right)<R_{1}$ for some $a_{n} \in[0, T]$. By (29), there exists $c_{n} \in[0, T]$ (without loss of generality, we assume $a_{n}<$ $c_{n}$.) such that $x_{n}\left(c_{n}\right)=M w\left(c_{n}\right)+R_{1}$ and $x_{n}(t) \leq M w(t)+R_{1}$ for $a_{n} \leq t \leq c_{n}$.

It can be checked that

$$
F_{n}\left(t, Z_{n}(t), Z_{n}^{\prime}(t)\right)>a(t) Z_{n}(t)+M+\bar{M}_{h},
$$

where $\bar{M}_{h}$ is defined by (15). have

In fact, if $t \in\left[a_{n}, c_{n}\right]$ is such that $1 / n \leq Z_{n}(t) \leq R_{1}$, we

$$
\begin{aligned}
F_{n}(t, & \left.Z_{n}(t), Z_{n}^{\prime}(t)\right) \\
& =F\left(t, Z_{n}(t), Z_{n}^{\prime}(t)\right) \\
& \geq a(t) Z_{n}(t)+\tilde{g}_{0}(x)>\max \left\{M+\bar{M}, \iota_{h} r\|\alpha\|_{1}\right\} \\
& \geq a(t) Z_{n}(t)+M+\bar{M} .
\end{aligned}
$$

and, if $t \in\left[a_{n}, c_{n}\right]$ is such that $Z_{n}(t) \leq 1 / n$, we have

$$
\begin{aligned}
F_{n}\left(t, Z_{n}(t), Z_{n}^{\prime}(t)\right) & =F\left(t, \frac{1}{n}, Z_{n}^{\prime}(t)\right) \\
& \geq \frac{a(t)}{n}+\tilde{g}_{0}\left(\frac{1}{n}\right)>\frac{a(t)}{n}+M+\bar{M}_{h} \\
& \geq a(t) Z_{n}(t)+M+\bar{M}_{h} .
\end{aligned}
$$

So (34) holds.

Using (17) (with $\lambda=1$ ) for $x_{n}(t)$ and the estimation (34), we have, for $t \in\left[a_{n}, c_{n}\right]$

$$
\begin{aligned}
Z_{n}^{\prime \prime}(t)= & -h(t) Z_{n}^{\prime}(t)-a(t) Z_{n}(t) \\
& -M+F_{n}\left(t, Z_{n}(t), Z_{n}^{\prime}(t)\right)+\frac{a(t)}{n} \\
> & -h(t) Z_{n}^{\prime}(t)-a(t) Z_{n}(t) \\
& -M+a(t) Z_{n}(t)+M+\bar{M}_{h}+\frac{a(t)}{n} \\
\geq & -\bar{M}_{h}-a(t) Z_{n}(t)-M \\
& +a(t) Z_{n}(t)+M+\bar{M}_{h}+\frac{a(t)}{n} \\
\geq & \frac{a(t)}{n} \geq 0 .
\end{aligned}
$$

As $Z_{n}^{\prime}\left(a_{n}\right)=0, Z_{n}^{\prime}(t)>0$ for all $t \in\left[a_{n}, c_{n}\right]$, so $Z_{n}(t)$ is strictly increasing on $\left[a_{n}, c_{n}\right]$. We use $\xi_{n}$ to denote the inverse function of $Z_{n}$ restricted to $\left[a_{n}, c_{n}\right]$.

Suppose that (27) does not hold, that is, for some $n \in N_{1}$, $Z_{n}(t)<1 / n<R_{1}$. Then there would exist $b_{n} \in\left(a_{n}, c_{n}\right)$ such that $Z_{n}\left(b_{n}\right)=1 / n$ and

$$
\begin{gathered}
Z_{n}(t) \leq \frac{1}{n} \quad \text { for } a_{n} \leq t \leq b_{n}, \\
\frac{1}{n} \leq Z_{n}(t) \leq R_{1} \quad \text { for } b_{n} \leq t \leq c_{n} .
\end{gathered}
$$

Multiplying (17) (with $\lambda=1$ ) by $Z_{n}^{\prime}(t)$ and integrating from $b_{n}$ to $c_{n}$, we obtain

$$
\begin{aligned}
& \int_{1 / n}^{R_{1}} F\left(\xi_{n}(Z), Z, Z^{\prime}\right) d Z \\
& \quad=\int_{b_{n}}^{c_{n}} F\left(t, Z_{n}(t), Z_{n}^{\prime}(t)\right) Z_{n}^{\prime}(t) d t \\
& =\int_{b_{n}}^{c_{n}} F_{n}\left(t, Z_{n}(t), Z_{n}^{\prime}(t)\right) Z_{n}^{\prime}(t) d t \\
& =\int_{b_{n}}^{c_{n}}\left(x_{n}^{\prime \prime}(t)+h(t) x_{n}^{\prime}(t)+a(t) x_{n}(t)-\frac{a(t)}{n}\right) Z_{n}^{\prime}(t) d t
\end{aligned}
$$




$$
\begin{aligned}
= & \int_{b_{n}}^{c_{n}} x_{n}^{\prime \prime}(t)\left(x_{n}^{\prime}(t)-M w^{\prime}(t)\right) d t+\int_{b_{n}}^{c_{n}} h(t) x_{n}^{\prime}(t) Z_{n}^{\prime}(t) d t \\
& +\int_{b_{n}}^{c_{n}}\left(a(t) x_{n}(t)-\frac{a(t)}{n}\right) Z_{n}^{\prime}(t) d t .
\end{aligned}
$$

By the facts $\left\|x_{n}\right\|<r,\left\|x_{n}^{\prime}\right\| \leq L r,\left\|w^{\prime}\right\| \leq r$ and the definition of $Z_{n}(t)$, we can obtain $\left|Z_{n}(t)\right| \leq r+T B,\left|Z_{n}^{\prime}(t)\right| \leq$ $(r+M) L$, together with $\left\|x_{n}\right\|<r$, implies that the second term and the third term are bounded. The first term is

$$
\begin{aligned}
& \frac{\left(\left[x_{n}^{\prime}\left(c_{n}\right)\right]^{2}-\left[x_{n}^{\prime}\left(b_{n}\right)\right]^{2}\right)}{2} \\
& \quad-M\left(x_{n}^{\prime}\left(c_{n}\right) w^{\prime}\left(c_{n}\right)-x_{n}^{\prime}\left(b_{n}\right) w^{\prime}\left(b_{n}\right)\right) \\
& \quad+M \int_{b_{n}}^{c_{n}} x_{n}^{\prime}(t) w^{\prime \prime}(t) d t,
\end{aligned}
$$

which is also bounded. As a consequence, there exists a $B_{1}>0$ such that

$$
\int_{1 / n}^{R_{1}} F\left(\xi_{n}(Z), Z, Z^{\prime}\right) d Z \leq B_{1} .
$$

On the other hand, by $\left(\mathrm{H}_{2}\right)$, we can choose $n_{2} \in N_{1}$ large enough such that

$$
\int_{1 / n}^{R_{1}} F\left(\xi_{n}(Z), Z, Z^{\prime}\right) d Z \geq \int_{1 / n}^{R_{1}} g_{0}(Z) d Z>B_{1}
$$

for all $n \in N_{2}=\left\{n_{2}, n_{2}+1, \ldots\right\}$. So (27) holds.

Furthermore, we can prove $Z_{n}(t)$ has a uniform positive lower bound $\delta$.

Lemma 6. There exist a constant $\delta>0$ such that, for all $n \in$ $\mathrm{N}_{2}$,

$$
Z_{n}(t) \geq \delta .
$$

Proof. Multiplying (17) (with $\lambda=1$ ) by $Z_{n}^{\prime}(t)$ and integrating from $a_{n}$ to $c_{n}$, we obtain

$$
\begin{aligned}
& \int_{\alpha_{n}}^{R_{1}} F\left(\xi_{n}(Z), Z, Z^{\prime}\right) d Z \\
& =\int_{a_{n}}^{c_{n}} F\left(t, Z_{n}(t), Z_{n}^{\prime}(t)\right) Z_{n}^{\prime}(t) d t \\
& =\int_{a_{n}}^{c_{n}} F_{n}\left(t, Z_{n}(t), Z_{n}^{\prime}(t)\right) Z_{n}^{\prime}(t) d t \\
& =\int_{a_{n}}^{c_{n}}\left(x_{n}^{\prime \prime}(t)+h(t) x_{n}^{\prime}(t)+a(t) x_{n}(t)-\frac{a(t)}{n}\right) Z_{n}^{\prime}(t) d t \\
& =\int_{a_{n}}^{c_{n}} x_{n}^{\prime \prime}(t)\left(x_{n}^{\prime}(t)-M w^{\prime}(t)\right) d t+\int_{a_{n}}^{c_{n}} h(t) x_{n}^{\prime}(t) Z_{n}^{\prime}(t) d t \\
& \quad+\int_{a_{n}}^{c_{n}}\left(a(t) x_{n}(t)-\frac{a(t)}{n}\right) Z_{n}^{\prime}(t) d t .
\end{aligned}
$$

In the same way as in the proof of (41), one way readily prove that the right-hand side of the above equality is bounded. On the other hand, if $n \in N_{2}$, by $\left(\mathrm{H}_{2}\right)$,

$$
\begin{aligned}
\int_{\alpha_{n}}^{R_{1}} F & \left(\xi_{n}(Z), Z, Z^{\prime}\right) d Z \\
& \geq \int_{\alpha_{n}}^{R_{1}} g_{0}(Z) d Z+M\left(R_{1}-\alpha_{n}\right) \longrightarrow+\infty
\end{aligned}
$$

if $\alpha_{n} \rightarrow 0_{+}$. Thus we know that there exists a constant $\delta>0$ such that $\alpha_{n} \geq \delta$. Hence (43) holds.

Next, we will prove (17) has periodic solution $x_{n}(t)$.

For $\iota r>0$, we can choose $n_{3} \in N_{2}$ such that $1 / n_{3}<\iota r$, which together with $\left(\mathrm{H}_{4}\right)$ imply

$$
w^{*} g\left(\iota r-M w^{*}\right)\left(1+\frac{h(r)}{g(r)}\right) \varrho((r+M) L)+\frac{1}{n_{3}}<r .
$$

Let $N_{3}=\left\{n_{3}, n_{3}+1, \ldots\right\}$. For $n \in N_{3}$, consider (17).

Next we claim that any fixed point $x_{n}$ of (18) for any $\lambda \epsilon$ $[0,1]$ must satisfy $\left\|x_{n}\right\| \neq r$. So, by using the Leray-Schauder alternative principle, (17) (with $\lambda=1$ ) has a periodic solution $x_{n}(t)$. Otherwise, assume that $x_{n}$ is a fixed point $x_{n}$ of (18) for some $\lambda \in[0,1]$ such that $\left\|x_{n}\right\|=r$. Note that

$$
\begin{aligned}
x_{n}(t)-\frac{1}{n} & =\lambda \int_{0}^{T} G(t, s) F_{n}\left(s, Z_{n}(s), Z_{n}^{\prime}(s)\right) d s \\
& \geq \lambda A \int_{0}^{T} F_{n}\left(s, Z_{n}(s), Z_{n}^{\prime}(s)\right) d s \\
& =\iota B \lambda \int_{0}^{T} F_{n}\left(s, Z_{n}(s), Z_{n}^{\prime}(s)\right) d s \\
& \geq \iota \max _{t \in[0, T]}\left\{\lambda \int_{0}^{T} G(t, s) F_{n}\left(s, Z_{n}(s), Z_{n}^{\prime}(s)\right) d s\right\} \\
& =\iota\left\|x_{n}-\frac{1}{n}\right\| .
\end{aligned}
$$

For $n \in N_{3}$, we have

$$
x_{n}(t) \geq \iota\left\|x_{n}-\frac{1}{n}\right\|+\frac{1}{n} \geq \iota\left(\left\|x_{n}\right\|-\frac{1}{n}\right)+\frac{1}{n} \geq \iota r .
$$

By (27) and assumption $\left(\mathrm{H}_{3}\right)$, for all $t \in[0, T]$ and $n \in N_{3}$, we have

$$
\begin{aligned}
& x_{n}(t) \\
& =\lambda \int_{0}^{T} G(t, s) F_{n}\left(s, Z_{n}(s), Z_{n}^{\prime}(s)\right) d s+\frac{1}{n} \\
& =\lambda \int_{0}^{T} G(t, s) F\left(s, Z_{n}(s), Z_{n}^{\prime}(s)\right) d s+\frac{1}{n}
\end{aligned}
$$




$$
\begin{aligned}
\leq & \int_{0}^{T} G(t, s) F\left(s, Z_{n}(s), Z_{n}^{\prime}(s)\right) d s+\frac{1}{n} \\
\leq & \int_{0}^{T} G(t, s)\left(g\left(Z_{n}(s)\right)+h\left(Z_{n}(s)\right)\right) \varrho\left(\left|Z_{n}^{\prime}(s)\right|\right) d s \\
& +\frac{1}{n} \\
\leq & \int_{0}^{T} G(t, s) g\left(Z_{n}(s)\right)\left(1+\frac{h\left(Z_{n}(s)\right)}{g\left(Z_{n}(s)\right)}\right) \varrho\left(\left|Z_{n}^{\prime}(s)\right|\right) d s \\
& +\frac{1}{n} \\
\leq & \int_{0}^{T} G(t, s) g\left(\iota r-M w^{*}\right)\left(1+\frac{h(r)}{g(r)}\right) \varrho((r+M) L) d s \\
& +\frac{1}{n} \\
\leq & g\left(\iota r-M w^{*}\right)\left(1+\frac{h(r)}{g(r)}\right) \varrho((r+M) L) w^{*}+\frac{1}{n_{3}} .
\end{aligned}
$$

Therefore,

$$
r=\|x\| \leq g\left(\iota r-M w^{*}\right)\left(1+\frac{h(r)}{g(r)}\right) \varrho((r+M) L) w^{*}+\frac{1}{n_{3}} .
$$

This is a contradiction to the choice of $n_{3}$ and the claim is proved.

The fact $\left\|x_{n}\right\|<r$ and $\left\|x_{n}^{\prime}(t)\right\|<\operatorname{Lr}$ show that $\left\{x_{n}\right\}_{n \in N_{3}}$ is a bounded and equicontinuous family on $[0, T]$. Now ArzelaAscoli Theorem guarantees that $\left\{x_{n}\right\}_{n \in N_{3}}$ has a subsequence $\left\{x_{n_{k}}\right\}_{k \in \mathbb{N}}$, converging uniformly on $[0, T]$ to a function $x \in$ $C[0, T]$. From the fact $\left\|x_{n}\right\|<r$ and $x_{n}(t)>\delta, x$ satisfies $\delta \leq x(t) \leq r$ for all $t$. Moreover, $\left\{x_{n_{k}}\right\}$ satisfies the integral equation

$$
x_{n_{k}}(t)=\int_{0}^{T} G(t, s) F\left(s, Z_{n_{k}}(s), Z_{n_{k}}^{\prime}(s)\right) d s+\frac{1}{n_{k}} .
$$

Letting $k \rightarrow \infty$, we arrive at

$$
x(t)=\int_{0}^{T} G(t, s) F\left(s, x(s)-M w(s), x^{\prime}(s)-M w^{\prime}(s)\right) d s,
$$

where the uniform continuity of $F(t, x, y)$ on $[0, T] \times[\delta, r] \times$ $[-(r+M) L,(r+M) L]$ is used. Therefore, $x$ is a positive periodic solution of (16) and $Z(t)=x(t)-M w(t) \geq \delta$. Thus we complete the prove of Theorem 4 .

Corollary 7. Let the nonlinearity in (1) be

$$
f(t, x, y)=\left(1+|y|^{\gamma}\right)\left(x^{-\alpha}+\mu x^{\beta}\right)+e(t),
$$

where $\alpha>1, \beta>0,1>\gamma \geq 0, \mu>0$ is a positive parameter, $e(t)$ is a T-periodic function. (i) If $\beta+\gamma<1$, then (1) has at least one positive periodic solution for each $\mu>0$.

(ii) If $\beta+\gamma \geq 1$, then (1) has at least one positive periodic solution for each $0<\mu<\mu_{1}$, where $\mu_{1}$ is some positive constant.

Proof. We will apply Theorem 4 with $M=\max _{0 \leq t \leq T}|e(t)|$ and $g(x)=x^{-\alpha}, h(x)=\mu x^{\beta}+2 M, \varrho(y)=1+|y|^{\gamma}$. Then condition $\left(\mathrm{H}_{1}\right)-\left(\mathrm{H}_{3}\right)$ are satisfied and existence condition $\left(\mathrm{H}_{4}\right)$ becomes

$$
\mu<\frac{r\left(\iota r-M w^{*}\right)^{\alpha}-w^{*}\left(1+(r+M)^{\gamma} L^{\gamma}\right)\left(1+2 M r^{\alpha}\right)}{w^{*}\left(1+(r+M)^{\gamma} L^{\gamma}\right) r^{\alpha+\beta}} .
$$

So (1) has at least one positive periodic solution for

$$
\begin{gathered}
0<\mu<\mu_{1} \\
=\sup _{r>M w^{*} / \iota}\left(r\left(\iota r-M w^{*}\right)^{\alpha}-w^{*}\left(1+(r+M)^{\gamma} L^{\gamma}\right)\right. \\
\left.\quad \times\left(1+2 M r^{\alpha}\right)\right) \\
\quad \times\left(w^{*}\left(1+(r+M)^{\gamma} L^{\gamma}\right) r^{\alpha+\beta}\right)^{-1} .
\end{gathered}
$$

Note that $\mu_{1}=\infty$ if $\beta+\gamma<1$ and $\mu_{1}<\infty$ if $\beta+\gamma \geq 1$. We have the desired results.

\section{Acknowledgments}

The research of X. Xing is supported by the Fund of the Key Disciplines in the General Colleges and Universities of Xin Jiang Uygur Autonomous Region (Grant no. 2012ZDKK13). It is a pleasure for the author to thank Professor J. Chu for his encouragement and helpful suggestions.

\section{References}

[1] J. L. Bravo and P. J. Torres, "Periodic solutions of a singular equation with indefinite weight," Advanced Nonlinear Studies, vol. 10, no. 4, pp. 927-938, 2010.

[2] J. Chu, P. J. Torres, and M. Zhang, "Periodic solutions of second order non-autonomous singular dynamical systems," Journal of Differential Equations, vol. 239, no. 1, pp. 196-212, 2007.

[3] D. Jiang, J. Chu, and M. Zhang, "Multiplicity of positive periodic solutions to superlinear repulsive singular equations," Journal of Differential Equations, vol. 211, no. 2, pp. 282-302, 2005.

[4] M. Meehan and D. O'Regan, "Existence theory for nonlinear Volterra integrodifferential and integral equations," Nonlinear Analysis: Theory, Methods \& Applications, vol. 31, no. 3-4, pp. 317-341, 1998.

[5] W. B. Gordon, "Conservative dynamical systems involving strong forces," Transactions of the American Mathematical Society, vol. 204, pp. 113-135, 1975.

[6] M. A. del Pino and R. F. Manásevich, "Infinitely many Tperiodic solutions for a problem arising in nonlinear elasticity," Journal of Differential Equations, vol. 103, no. 2, pp. 260-277, 1993. 
[7] M. Zhang, "A relationship between the periodic and the Dirichlet BVPs of singular differential equations," Proceedings of the Royal Society of Edinburgh A, vol. 128, no. 5, pp. 1099-1114, 1998.

[8] J. Chu and P. J. Torres, "Applications of Schauder's fixed point theorem to singular differential equations," Bulletin of the London Mathematical Society, vol. 39, no. 4, pp. 653-660, 2007.

[9] D. Franco and P. J. Torres, "Periodic solutions of singular systems without the strong force condition," Proceedings of the American Mathematical Society, vol. 136, no. 4, pp. 1229-1236, 2008.

[10] D. Bonheure and C. de Coster, "Forced singular oscillators and the method of lower and upper solutions," Topological Methods in Nonlinear Analysis, vol. 22, no. 2, pp. 297-317, 2003.

[11] M. Zhang, "Periodic solutions of equations of Emarkov-Pinney type," Advanced Nonlinear Studies, vol. 6, no. 1, pp. 57-67, 2006.

[12] P. J. Torres, "Existence of one-signed periodic solutions of some second-order differential equations via a Krasnoselskii fixed point theorem," Journal of Differential Equations, vol. 190, no. 2, pp. 643-662, 2003.

[13] P. J. Torres, "Existence and stability of periodic solutions for second-order semilinear differential equations with a singular nonlinearity," Proceedings of the Royal Society of Edinburgh A, vol. 137, no. 1, pp. 195-201, 2007.

[14] J. Chu, N. Fan, and P. J. Torres, "Periodic solutions for second order singular damped differential equations," Journal of Mathematical Analysis and Applications, vol. 388, no. 2, pp. 665-675, 2012.

[15] J. Chu and M. Li, "Positive periodic solutions of Hill's equations with singular nonlinear perturbations," Nonlinear Analysis: Theory, Methods \& Applications, vol. 69, no. 1, pp. 276-286, 2008.

[16] X. Li and Z. Zhang, "Periodic solutions for damped differential equations with a weak repulsive singularity," Nonlinear Analysis: Theory, Methods \& Applications, vol. 70, no. 6, pp. 2395-2399, 2009.

[17] M. Zhang, "Periodic solutions of damped differential systems with repulsive singular forces," Proceedings of the American Mathematical Society, vol. 127, no. 2, pp. 401-407, 1999.

[18] R. Hakl and P. J. Torres, "Maximum and antimaximum principles for a second order differential operator with variable coefficients of indefinite sign," Applied Mathematics and Computation, vol. 217, no. 19, pp. 7599-7611, 2011.

[19] M. Zhang, "Optimal conditions for maximum and antimaximum principles of the periodic solution problem," Boundary Value Problems, vol. 2010, Article ID 410986, 26 pages, 2010.

[20] A. Granas, R. B. Guenther, and J. W. Lee, "Some general existence principles in the Carathéodory theory of nonlinear differential systems," Journal de Mathématiques Pures et Appliquées, vol. 70, no. 2, pp. 153-196, 1991. 


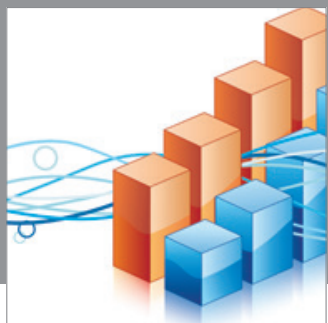

Advances in

Operations Research

mansans

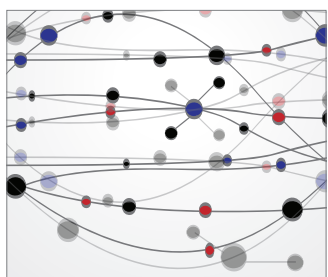

The Scientific World Journal
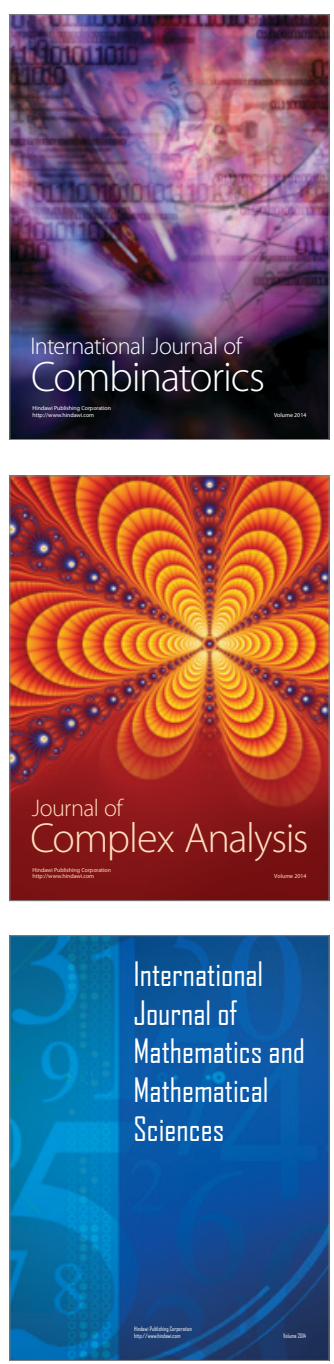
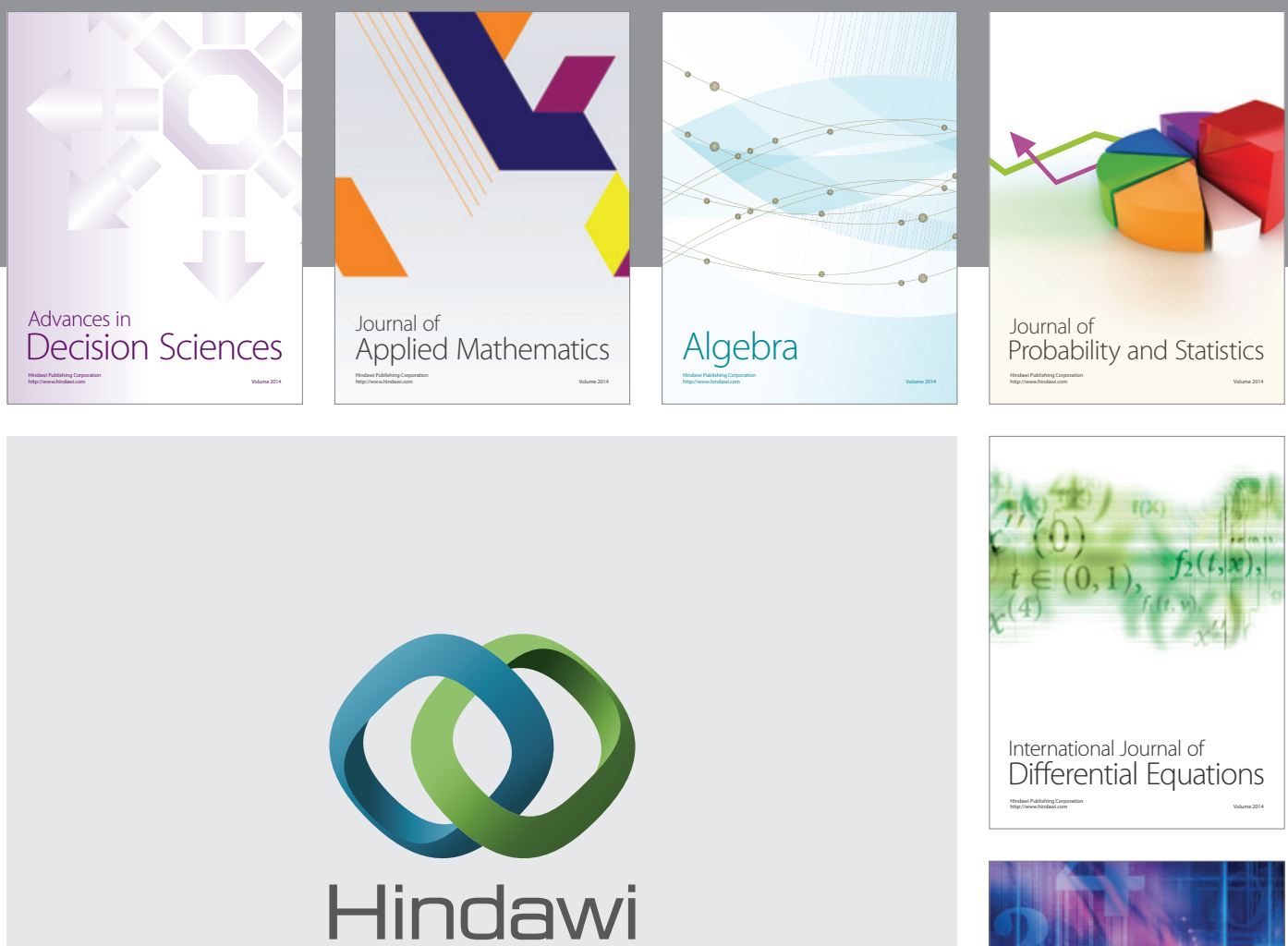

Submit your manuscripts at http://www.hindawi.com
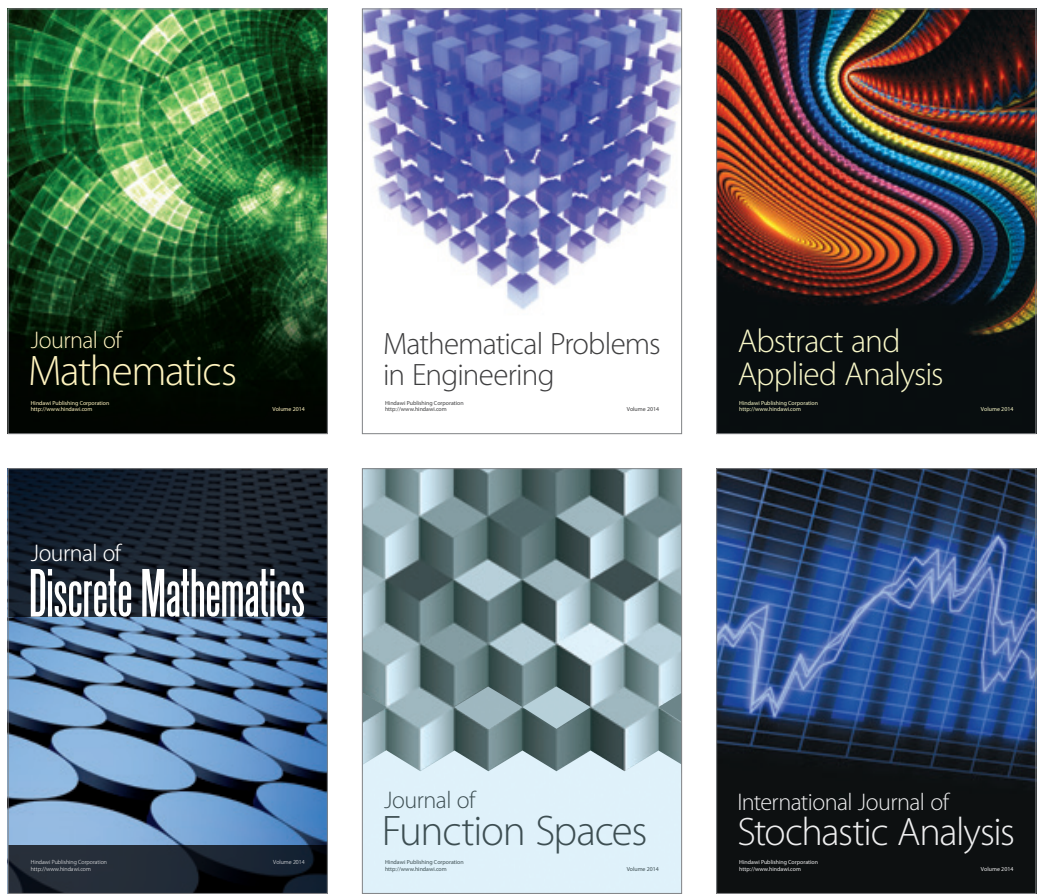

Journal of

Function Spaces

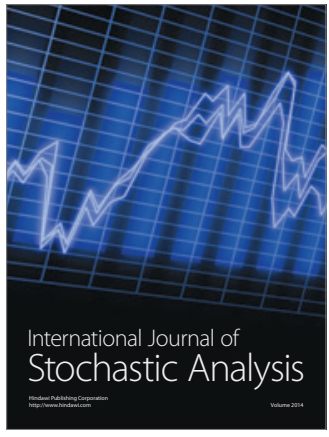

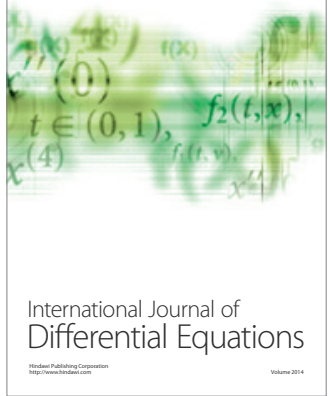
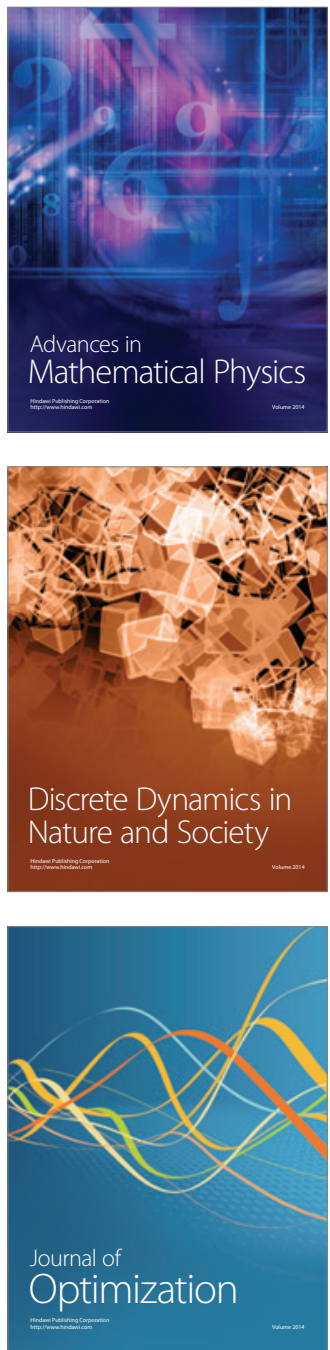